\title{
Automated tests of ANA immunofluorescence as throughput autoantibody detection technology: strengths and limitations
}

Pier Luigi Meroni ${ }^{1,2,5^{*}}$, Nicola Bizzaro ${ }^{3}$, Ilaria Cavazzana ${ }^{4}$, Maria Orietta Borghi ${ }^{1,2}$ and Angela Tincani ${ }^{4}$

\begin{abstract}
Anti-nuclear antibody (ANA) assay is a screening test used for almost all autoimmune rheumatic diseases, and in a number of these cases, it is a diagnostic/classification parameter. In addition, ANA is also a useful test for additional autoimmune disorders. The indirect immunofluorescence technique on monolayers of cultured epithelial cells is the current recommended method because it has higher sensitivity than solid phase assays. However, the technique is time-consuming and requires skilled operators. Automated ANA reading systems have recently been developed, which offer the advantage of faster and much easier performance as well as better harmonization in the interpretation of the results. Preliminary validation studies of these systems have given promising results in terms of analytical specificity and reproducibility. However, these techniques require further validation in clinical studies and need improvement in their recognition of mixed or less common staining patterns.
\end{abstract}

Keywords: Anti-nuclear antibodies, Indirect immunofluorescence, Autoimmunity

\section{Background}

Anti-nuclear antibody assay (ANA) is the screening test of choice for diagnosis of almost all systemic autoimmune rheumatic diseases (SARDs) because of its greater sensitivity compared with other assays, even though its specificity is much lower (Box 1) [1]. The gold standard method for ANA detection is still indirect immunofluorescence (IIF) on human epithelial (HEp-2) cells, as the alternative tests cannot display comparable sensitivity [2]. However, the technique is time-consuming and requires skilled operators. This fact together with the widespread increase in ANA requests and the reduction of laboratory facilities because of the budget constriction generated a strong need for advanced automated platforms as in other branches of the laboratory medicine.

\section{ANA automated reading systems}

Currently, at least six commercial systems for the automated reading of ANA IIF are available: Aklides

\footnotetext{
* Correspondence: pierluigi.meroni@unimi.it

'Department of Clinical Sciences \& Community Health, University of Milan, Milan, Italy

${ }^{2}$ Istituto Auxologico Italiano, Milan, Italy

Full list of author information is available at the end of the article
}

(Medipan, Dahlewitz, Germany), EUROPattern (Euroimmun AG, Luebeck, Germany), Helios (Aesku Diagnostics, Wendelsheim, Germany), Image Navigator (ImmunoConcepts, Sacramento, CA), NOVA View (Inova Diagnostics, San Diego, CA), and Zenit G-Sight (A. Menarini Diagnostics, Florence, Italy).

These systems are based on a composition of different hardware modules combined with mathematical patternrecognition software algorithms, enabling fully automated image acquisition, analysis, and evaluation of IIF ANA tests.

Samples can be classified as positive or negative and the main IIF pattern recognized (Table 1). In addition, quantitative fluorescence intensity value (equivalent to the end-point titer) can be obtained. To date, 13 studies have been published assessing the reliability of automated IIF analysis as a standardized alternative for the conventional manual visual approach (Table 2) [3-14].

The reported advantages of these systems include reduction in intra-laboratory and inter-laboratory variability, improvement in correlation between staining patterns with corresponding autoantibody reactivities, higher throughput in laboratory workflows, no requirement for a 


Box 1 Anti-nuclear antibody assay
Best screening test for SLE
- Sensitivity $\geq 95 \%$
- Specificity is only $57 \%$ for SLE compared with related
Key diagnostic assay for:
- SSc (sensitivity $85 \%$ )
- SS (sensitivity $48 \%$ )
- Drug-induced lupus (sensitivity 100\%)
- PM/DM (sensitivity $61 \%$ )
- JIA (sensitivity $57 \%$ )
- MCTD (sensitivity 100\%)
- Autoimmune hepatitis (sensitivity up to 60\%)
Important role in assessing prognosis in Raynaud's
phenomenon [2].
DM, dermatomyositis; JIA, juvenile idiopathic arthritis; MCTD,
mixed connective tissue disease; PM, polymyositis; SLE, systemic
lupus erythematosus; SSc, systemic sclerosis; SS, Sjögren's

darkroom, integrated file storage, and easy retrieval of scanned wells.

\section{Comparison of the available ANA automated reading systems}

Although comparable performance between automated and conventional ANA IIF analysis for the interpretation of negative and positive samples has been reported, discrepancies between patterns have been found, especially when systems are able to detect basic patterns

Table 1 Types of indirect immunofluorescence pattern identified by the currently available automated systems for anti-nuclear antibody assay

\begin{tabular}{|c|c|}
\hline System & Pattern \\
\hline Aklides & $\begin{array}{l}\text { Homogeneous, speckled, nucleolar, centromeric, } \\
\text { nuclear dots, cytoplasmic }\end{array}$ \\
\hline EuroPattern & $\begin{array}{l}\text { Homogeneous, speckled, nucleolar, centromeric, } \\
\text { nuclear dots, cytoplasmic }\end{array}$ \\
\hline Helios & Visual recognition by the operator \\
\hline Image Navigator & Visual recognition by the operator \\
\hline Nova View & $\begin{array}{l}\text { Homogeneous, speckled, nucleolar, centromeric, } \\
\text { nuclear dots, cytoplasmic }\end{array}$ \\
\hline Zenit G-Sight & $\begin{array}{l}\text { Homogeneous, speckled, nucleolar, centromeric, } \\
\text { nuclear dots, mitochondrial }\end{array}$ \\
\hline
\end{tabular}

Table 2 Automated/manual positive-negative agreement (PNA) for each anti-nuclear antibody indirect immunofluorescence reading system, based on 13 published studies

\begin{tabular}{llll}
\hline System & Studies, $\mathbf{n}$ & Patients, $\mathbf{n}$ & PNA, mean \\
\hline Aklides & 3 & 1801 & 0.95 \\
EuroPattern & 2 & 467 & 0.97 \\
Helios & 1 & 1005 & 0.98 \\
Image Navigator & 1 & 3185 & 0.99 \\
Nova View & 2 & 842 & 0.95 \\
Zenit G-Sight & 3 & 830 & 0.92 \\
All systems & 1 & 149 & 0.96 \\
Total & 13 & 8279 & 0.97 \\
\hline
\end{tabular}

only, or when mixed fluorescent patterns are present in the samples [3-14].

Some automated IIF systems present misinterpretation difficulties when antibodies react with a limited and specific cell component, such as Golgi apparatus, nuclear dots, or nuclear membrane [3-14]. Such misinterpretation may have implications in clinical settings, emphasizing the need and importance of visual validation (Table 3).

Such IIF assays have identifed more than 50 autoantibodies against 30 different nuclear and cytoplasmic antigens [16]. The use of large cultured cells with high rates of mitosis enables adequate pattern recognition by evaluation of the fluorescence distribution during different phases of the cell cycle. In fact, identification of cell cycle dynamics (for example, interphase, mitosis) is crucial both for defining different patterns (such as the fine or large speckled patterns within a speckled staining pattern, the centromere patterns and the PCNA patterns) and for distinguishing between different patterns (for example anti-nuclear membrane from the homogeneous pattern).

Correct identification of different IIF patterns is sometimes diagnostic (for example, the centromere pattern and the PCNA pattern) or may suggest the occurrence of autoantibodies to specific antigens (Table 3). Many sera contain more than one antibody; in such cases, accurate analysis of the different patterns often requires direct evaluation of the slides to enable exact definition of the autoantibody profile in a given patient.

Systemic sclerosis (SSc) represents a paradigmatic example of an autoimmune disease that is characterized by the occurrence of ANA in virtually all patients, but for which interpretation of the patterns is complesx [17]. In fact, SSc ANA are mainly represented by four mutually exclusive specificities: anti-centromere (ACA), antitopoisomerase I, anti-nucleolar, and anti-RNA polymerase III antibodies. Anti-PM-Scl, U1-RNP and anti-Ku are 
Table 3 Indirect immunofluorescence patterns detected on HEp-2 cells, with, related antigens and diagnosis ${ }^{a}$

\begin{tabular}{|c|c|c|}
\hline & Related antigens & Related diagnosis \\
\hline \multicolumn{3}{|l|}{ Nuclear patterns } \\
\hline Homogeneous & DNA, histones, chromatin/nucleosomes & SLE, drug-induced SLE, JIA \\
\hline Peripheral/rim or nuclear envelope & $\begin{array}{l}\text { Lamins, LAP1/2 gp210, nucleoporin p62; Tpr nuclear } \\
\text { envelope and nuclear pore complex antigens }\end{array}$ & $\begin{array}{l}\text { SLE, RA, PBC, myositis, autoimmune liver disease, } \\
\text { PAPS }\end{array}$ \\
\hline Coarse speckled & U1-snRNP, U2-6 snRNP (Sm), nuclear matrix & MCTD, SLE, Raynaud, SSc, SS, UCTD \\
\hline Fine speckled & SSA/Ro, SSB/La, common to many antigens & SLE, SS, SSC, myositis, MCTD \\
\hline Dense fine speckled & DFS70/LEDGF-P75 & $\begin{array}{l}\text { Healthy subjects and other inflammatory } \\
\text { conditions }\end{array}$ \\
\hline PCNA & $\begin{array}{l}\text { Auxiliary protein proliferating cell nuclear antigen: } \\
\text { elongation factor of DNA polymerase } \delta\end{array}$ & SLE, lymphoproliferative diseases, SS \\
\hline Diffuse speckled with "cloudy" mitoses & Topoisomerase-I & SSC \\
\hline Centromere & Kinetochore: CENP-A, CENP-B, CENP-C, CENP-F & SSC (limited) \\
\hline Nucleolar homogeneous & $\begin{array}{l}\text { PM/Scl, RNA polymerase, To/Th , B23 phosphoprotein/ } \\
\text { numatrin }\end{array}$ & SSc, myositis, overlap myositis/SSc \\
\hline Nucleolar speckled & RNA polymerase (I to III) & SSC \\
\hline Nucleolar clumpy & U3-RNP (fibrillarin) & SSC \\
\hline Multiple/few nuclear dots & $\begin{array}{l}\text { Sp100/140, PML bodies, NDP53, p80-coilin, PML } \\
\text { bodies }\end{array}$ & $\mathrm{PBC}, \mathrm{CAH}, \mathrm{SS}$ \\
\hline $\begin{array}{l}\text { Centrosome/centriole (formerly spindle } \\
\text { apparatus) }\end{array}$ & Enolase, ninein, pericentrin & $\begin{array}{l}\text { SSc, Raynaud's phenomenon, inflammatory } \\
\text { disease }\end{array}$ \\
\hline MSA & NuMA/centrophilin Hseg5 & $\begin{array}{l}\text { RA, inflammatory conditions; pneumonia } \\
\text { (mycoplasma) }\end{array}$ \\
\hline \multicolumn{3}{|l|}{ Cytoplasmic patterns } \\
\hline $\begin{array}{l}\text { Diffuse homogeneous (nucleoli } \\
\text { positive) }\end{array}$ & Ribosomal proteins & SLE \\
\hline Fine speckled & Jo-1, SRP, PDH (mitochondria) & Myositides, DM, PBC, interstitial lung disease \\
\hline Discrete speckled & $\begin{array}{l}\text { Endosome (early endosome antigen 1), GW/P bodies, } \\
\text { multivesicular bodies/lysosomes }\end{array}$ & Neurological conditions, SS, SLE, RA, PBC \\
\hline Golgi complex & Golgi proteins & $\begin{array}{l}\text { SLE, SS, RA, overlap syndromes, cerebellar } \\
\text { ataxia }\end{array}$ \\
\hline Cytoplasmic fibers & Actin, cytokeratin, tropomyosin, vimentin & $\begin{array}{l}\mathrm{CAH}, \mathrm{DM} \text {, infections and other inflammatory } \\
\text { diseases }\end{array}$ \\
\hline
\end{tabular}

CAH, chronic autoimmune hepatitis; CENP, centromere protein; DM, dermatomyositis; DFS70/LEDGF, dense fine speckled/lens epithelium-derived growth factor; $J \mathrm{~A}$, juvenile idiopathic arthritis; MCTD, mixed connective tissue disease; MSA, mitotic spindle apparatus; PAPS, primary antiphospholipid syndrome; PBC, primary biliary cirrhosis; PCNA, proliferating cell nuclear antigen; PDH, phosphate dehydrogenase; PM, polymyositis; RA, rheumatoid arthritis; Scl, scleroderma; SLE, systemic lupus erythematosus; snRNP, small nuclear ribonuclear protein; SRP, signal recognition particle; SSc, systemic sclerosis; SS, Sjögren's syndrome; UCTD, undifferentiated connective tissue disease.

${ }^{a}$ Modified from Agmon-Levin et al. [15].

usually detected in overlap syndromes. About $60 \%$ of patients with SSc have ACA or anti-topoisomerase I antibodies as disease markers. Many other ANA that are present in SSc (for example, anti-RNA polymerase III, anti-Th/To, anti-PM/Scl, anti-Ku, anti-fibrillarin) are directed against different proteins localized in the nucleus and nucleolus. These antigen-antibody systems identify SSc subgroups with different evolution, organ involvement, and survival prognosis. The use of IIF for detection of ANA is mandatory for SSc diagnosis, displaying a sensitivity of $85 \%$ [1]. ACA and anti-topoisomerase I negative sera show strong anti-nuclear staining, featuring speckled or nucleolar (homogeneous, clumpy or speckled) patterns (Box 1). Therefore, the definition of the single nucleolar staining could address the suspect of specific autoantibodies, relevant for the diagnosis of SSc. A nucleolar ANA associated with new onset of Raynaud's phenomenon could be helpful in identifying a patient with early disease, sometimes associated with severe organ involvement. It is essential that ANA results are confirmed by more specific methods such as western blotting or immunoprecipitation assays.

All these points underline the importance of correct interpretation of a given fluorescence pattern, and the need for standardization of analysis in automated systems.

There is one other important point about using automated systems for ANA reading. The ANA test was originally ordered predominantly by rheumatologists and 
clinical immunologists, but nowadays a broader range of clinical disciplines (including primary care, dermatology, nephrology, gastroenterology, neurology, oncology, hematology, obstetrics, gynecology, cardiology) are currently ordering the test. This change in test referral patterns affects the post-test probability for a given disease, as screening tests with limited specificity (such as IIF ANA) are strongly affected when the pre-test probability in a given population decreases [17]. A positive ANA test obtained outside of the rheumatologic setting displays poor predictive value for future development of a rheumatic disease, but it represents a significant risk factor for SLE. Taking into account that the prevalence of SLE is 1 in $2000(0.05 \%)$, the observed frequency of $2.5 \%$ in individuals with a 1/80 positive ANA test represents a 50-fold relative risk for development of the disease $[18,19]$. Thus, ANA testing is a useful tool for SLE diagnosis.

\section{Conclusions}

Current evidence from preliminary study results indicates that there is good correlation between manual and automated interpretation of ANA IIF assays, at least in the ability to discriminate between positive and negative results and in recognizing the main IIF patterns. Such systems will therefore speed up routine performance of these tests and help to harmonize interpretation of the results across laboratories. However, there is a need to have their clinical diagnostic power validated by clinical studies, in addition to the analytical studies that have already been published. In addition, these new systems could be further improved if they were better able to recognize mixed fluorescent or less common fluorescent patterns.

\section{Abbreviations \\ ACA: Anti-centromere antibodies; ANA: Anti-nuclear antibody; $\mathrm{CAH}$ : Chronic autoimmune hepatitis; CENP: Centromere protein; DFS70/LEDGF: Dense fine speckled/lens epithelium-derived growth factor; DM: Dermatomyositis; IIF: Indirect immunofluorescence; JIA: Juvenile idiopathic arthritis; MCTD: Mixed connective tissue disease; MSA: mitotic spindle apparatus; PAPS: Primary antiphospholipid syndrome; PBC: Primary biliary cirrhosis; PCNA: Proliferating cell nuclear antigen; $\mathrm{PDH}$ : Phosphate dehydrogenase; PM-Scl: Polymyositis-scleroderma; PM: Polymyositis; PNA: Positive/negative agreement; RA: Rheumatoid arthritis; SARDS: Systemic autoimmune rheumatic disease; SLE: Systemic lupus erythematosus; snRNP: Small nuclear ribonuclear protein; SSc: Systemic sclerosis; SS: Sjögren's syndrome; UCTD: Undifferentiated connective tissue disease.}

\section{Competing interests}

PM has received fees as consultant for Inova and from BioRad; NB has been a paid consultant to Inova Diagnostics and has received lecture fees from A Menarini Diagnostics; and AT has received funding and reimbursements from IL, BioRad, and Thermofisher.

\section{Author contributions}

All authors contributed equally to conception, design, acquisition of data, and analysis/interpretation of data; all have been involved in drafting and revising the manuscript; all have given final approval of the version to be published; and all agree to be accountable for all aspects of the work. All authors read and approved the final manuscript.

\section{Author information}

PLM is Professor of Rheumatology at the University of Milan. MOB is deputy Director of the Experimental Immuno-Rheumatology Laboratory at the Istituto Auxologico Italiano. NB is Director of the Diagnostic Department at San Antonio Hospital, Tolmezzo. AT is Professor of Rheumatology at the University of Brescia and head of Rheumatology and Clinical Immunology Unit at Spedali Civili of Brescia. IC has a tenured position in Rheumatology and Clinical Immunology Unit at Spedali Civili of Brescia.

\section{Author details}

'Department of Clinical Sciences \& Community Health, University of Milan, Milan, Italy. ${ }^{2}$ Istituto Auxologico Italiano, Milan, Italy. ${ }^{3}$ Laboratory of Clinical Pathology, Ospedale San Antonio, Tolmezzo, Italy. ${ }^{4}$ Rheumatology Unit and Chair, Spedali Civili di Brescia, Università degli Studi di Brescia, Brescia, Italy. ${ }^{5}$ Division of Rheumatology, Istituto G. Pini, Piazza C. Ferrari, 1, 20122 Milan, Italy.

Received: 10 February 2014 Accepted: 10 February 2014 Published: 03 Mar 2014

\section{References}

1. Solomon DH, Kavanaugh AJ, Schur PH: American College Of Rheumatology Ad Hoc Committee on immunologic testing guidelines. Evidence-based guidelines for the use of immunologic tests: antinuclear antibody testing. Arthritis Rheum 2002, 47:434-444.

2. Meroni PL, Schur PH: ANA screening: an old test with new recommendations. Ann Rheum Dis 2010, 69:1420-1422.

3. Egerer K, Roggenbuck D, Hiemann R, Weyer MG, Büttner T, Radau B, Krause R, Lehmann B, Feist E, Burmester GR: Automated evaluation of autoantibodies on human epithelial-2 cells as an approach to standardize cell-based immunofluorescence tests. Arthritis Res Ther 2010, 12:R40.

4. Voigt J, Krause C, Rohwäder E, Saschenbrecker S, Hahn M, Danckwardt M, Feirer C, Ens K, Fechner K, Barth E, Martinetz T, Stöcker W: Automated indirect immunofluorescence evaluation of antinuclear autoantibodies on HEp-2 cells. Clin Dev Immunol 2012, 2012:651058.

5. Kivity S, Gilburd B, Agmon-Levin N, Carrasco MG, Tzaffir Y, Sofer Y, Mandel M, Buttner T, Roggenbuck D, Matucci-Cerinic M, Danko K, Hoyos ML, Shoenfeld Y: A novel automated indirect immunofluorescence autoantibody evaluation. Clin Rheumatol 2012, 31:503-9.

6. Melegari A, Bonaguri C, Russo A, Luisita B, Trenti T, Lippi G: A comparative study on the reliability of an automated system for the evaluation of cell-based indirect immunofluorescence. Autoimmun Rev 2012, 11:713-716.

7. Bossuyt X, Cooreman S, De Baere H, Verschueren P, Westhovens R, Blockmans D, Mariën G: Detection of antinuclear antibodies by automated indirect immunofluorescence analysis. Clin Chim Acta 2013, 415:101-6.

8. Copple SS, Giles RS, Jaskowski TD, Hill HR: Comparison of nova view ${ }^{T M}$ antinuclear antibody titers to manual fluorescent microscopy. In Proceedings of the 8th International Congress on Autoimmunity, Granada (Spain), May 9-13, 2012. Abstract n. 300 http://www.kenes.com/ autoimmunity2012\%.

9. Krieger T: ANA detection with an automated reading system. Does it work? In Proceedings of the 8th International Congress on Autoimmunity, Granada (Spain), May 9-13, 2012. Abstract n. 647 http://www2.kenes.com/ autoimmunity2012/Pages/Home.aspx.

10. Matthias T: Development and validation of an IFA processor with integrated optical system. In Proceedings of the 8th International Congress on Autoimmunity, Granada (Spain), May 9-13, 2012. Abstract n. 303 http:// www2.kenes.com/autoimmunity2012/Pages/Home.aspx.

11. Vercammen MJ, De Roeck K, Meirlaen P: Antinuclear antibody (ANA) Screening, titration and pattern recognition: performance of the Zenit G-Sight automatic system. In Proceedings of the 8th International Congress on Autoimmunity, Granada (Spain), May 9-13, 2012. Abstract n. 1014 http://www2.kenes.com/autoimmunity2012/Pages/Home.aspx.

12. Hoy E, Stoeckl B: Automated fluorescent microscopy shows strong correlation with conventional reading of anti-nuclear antibody tests. In Proceedings of the 2nd International Congress on Controversies in Rheumatology \& Autoimmunity (CORA), Budapest (Hungary),April 4-6, 2013. Abstract n. 443 http://www.kenes.com/cora/. 
13. Bonroy C, Verfaillie C, Smith V, Persijn L, De Witte E, De Keyser F, Devreese K: Automated indirect immunofluorescence antinuclear antibody analysis is a standardized alternative for visual microscope interpretation. Clin Chem Lab Med 2013, 51:1771-9.

14. Bizzaro N, Antico A, Platzgummer S, Tonutti E, Bassetti D, Pesente F, Tozzoli R, Tampoia M, Villalta D, Study Group on Autoimmune Diseases of the Italian Society of Laboratory Medicine, Italy: Automated antinuclear immunofluorescence antibody screening: a comparative study of six computer-aided diagnostic systems. Autoimmun Rev 2014, 13:292-8.

15. Agmon-Levin N, Damoiseaux J, Kallenberg C, Sack U, Witte T, Herold M, Bossuyt X, Musset L, Cervera R, Plaza-Lopez A, Dias C, Sousa MJ, Radice A, Eriksson C, Hultgren O, Viander M, Khamashta M, Regenass S, Andrade LE, Wiik A, Tincani A, Rönnelid J, Bloch DB, Fritzler MJ, Chan EK, Garcia-De La Torre I, Konstantinov KN, Lahita R, Wilson M, Vainio O, et al: International recommendations for the assessment of autoantibodies to cellular antigens referred to as anti-nuclear antibodies. Ann Rheum Dis 2014, 73:17-23.

16. Burlingame R, Peebles C: Detection of antibodies. In Autoantibodies and autoimmunity: molecular mechanisms in health and disease. Edited by Pollard KM. Weinheim, Germany: Wiley VCH; 2006:88.

17. Koenig $M$, Dieudé $M$, Senécal JL: Predictive value of antinuclear autoantibodies: the lessons of the systemic sclerosis autoantibodies. Autoimmun Rev 2008, 7:588-593.

18. Abeles $\mathrm{AM}$, Abeles $\mathrm{M}$ : The clinical utility of a positive antinuclear antibody test result. Am J Med 2013, 126:342-348.

19. Meroni PL, Chan EKL, Tincani A, Garcia de la Torre I, Andrade ELC: Antinuclear antibody test: when to order? Am J Med 2013, 126:e17.

10.1186/1741-7015-12-38

Cite this article as: Meroni et al: Automated tests of ANA immunofluorescence as throughput autoantibody detection technology: strengths and limitations. BMC Medicine 2014, 12:38

\section{Submit your next manuscript to BioMed Central and take full advantage of:}

- Convenient online submission

- Thorough peer review

- No space constraints or color figure charges

- Immediate publication on acceptance

- Inclusion in PubMed, CAS, Scopus and Google Scholar

- Research which is freely available for redistribution 